\title{
Precessing microblazars and unidentified gamma-ray sources
}

\author{
M. M. Kaufman Bernadó ${ }^{1}$, G. E. Romero ${ }^{1, \star}$, and I. F. Mirabel ${ }^{2,3, \star}$ \\ 1 Instituto Argentino de Radioastronomía, C.C.5, (1894) Villa Elisa, Buenos Aires, Argentina \\ 2 CEA/DMS/DAPNIA/Service D'Astrophysique, Centre d'Etudes de Saclay, 91191 Gif-sur-Yvette, France \\ 3 Instituto de Astronomía y Física del Espacio/CONICET, C.C. 67, Suc. 28, Buenos Aires, Argentina
}

Received 22 January 2002 / Accepted 13 February 2002

\begin{abstract}
The recent discovery by Paredes et al. (2000) of a persistent microquasar that is positionally coincident with an unidentified gamma-ray source has open the possibility that other sources in the Third EGRET Catalog could be interpreted as microquasars as well. In this letter we show that some variable unidentified EGRET sources in the galactic plane could be produced by faint, otherwise undetected microquasars with precessing jets. When the jet points towards the observer, gamma-ray emission resulting from upscattered stellar photons could be detectable yielding a variable source with weak or undetectable counterpart at longer wavelengths. Strategies for detecting these "microblazars" with forthcoming satellites are briefly discussed.
\end{abstract}

Key words. X-ray binaries - stars: early-type - gamma-rays: observations - gamma-rays: theory

\section{Introduction}

The third and final EGRET catalog (Hartman et al. 1999) lists about 170 gamma-ray sources not yet clearly identified with objects detected at lower energies. Many of these sources concentrate towards the galactic plane and correlate with the spiral arms of the Galaxy, indicating a significant contribution from Population I objects (Romero et al. 1999; Romero 2001). From a purely statistical point of view, at least two different populations can be identified: one of them possibly associated with the Gould belt, a nearby star-forming region at $\sim 600 \mathrm{pc}$, and the other formed by brighter sources found at lower latitudes (Gehrels et al. 2000; Grenier 2000).

Several types of objects have been proposed as possible counterparts of galactic gamma-ray sources, including early-type stars (both isolated and forming binary systems), accreting neutron stars, radio-quiet pulsars, interacting supernova remnants, and black hole candidates (see Romero 2001 and references therein). Very recently, Paredes et al. (2000), Grenier (2001) and Romero (2001) have suggested that microquasars can also be responsible for some unidentified sources. In particular, Paredes et al. (2000) have proposed that the microquasar LS 5039, a massive X-ray binary with persistent non-thermal radio emission, is physically associated with the gamma-ray source 3EG J1824-1514. They suggested that the observed gamma-ray flux is the result of external Compton scattering of UV photons from the high-mass stellar companion.

Send offprint requests to: M. M. Kaufman Bernadó, e-mail: mar@vianw.com.ar

* Member of CONICET.
The fact that there is a significant number of variable gamma-ray sources at low galactic latitudes (e.g. Torres et al. 2001; Romero et al. 2001) along with the steady properties of gamma-ray pulsars and interacting supernova remnants, makes the idea of gamma-ray emitting microquasars particularly attractive. Mirabel \& Rodríguez (1999) proposed that microquasars with jets forming a small angle with the line of sight - by analogy with the unified model for AGNs - could appear as microblazars, namely, as sources with highly variable and enhanced nonthermal flux due to Doppler boosting.

In this letter we shall present a model for galactic variable gamma-ray sources based on the idea of precessing microblazars. In our model, the jet precession will be induced by the gravitational torque of the stellar companion, which is in a non-coplanar orbit, on the accretion disk of the primary (usually assumed to be a stellar black hole). Gamma-rays can be produced by external Compton scattering of UV stellar photons of the massive companions by relativistic leptons (electrons and/or positrons) far from the base of the jet. We shall discuss cases where the particles are in the form of a "blob" ejected from the central engine and where the particles are continuously injected in the form of a persistent jet. In the case of highmass binaries, this mechanism for generation of gammarays is more efficient than Compton scattering upon disk photons or self-synchrotron jet emission (Georganopoulos et al. 2001a) and, in combination with the gravitational effects of the stellar companion, can render suitable variable gamma-ray sources. We shall briefly discuss how these sources can be identified in the light of forthcoming satellite experiments like INTEGRAL. Although microblazars 
have been previously considered in the literature (e.g. Aharonian \& Atoyan 1998; Mirabel \& Rodríguez 1999; Georganopoulos et al. 2001a), this is the first detailed discussion in terms of unidentified gamma-ray sources.

\section{Variable gamma-ray emission from microblazars}

Let us consider a high-mass binary where accretion onto the compact object (a black hole) results in the production of twin, relativistic $\mathrm{e}^{+} \mathrm{e}^{-}$-pair jets propagating in opposite directions. A sudden variation in the injection rate can result in the formation of a blob (for which we adopt spherical geometry) which propagates down the jet with a bulk Lorentz factor $\Gamma$. We shall follow the general treatment derived by Georganopoulos et al. (2001b) for external Compton scattering in extragalactic blazars, adapting it to microblazars. In the blob frame the relativistic leptons are considered to have an isotropic power-law density distribution $n^{\prime}\left(\gamma^{\prime}\right)=(k / 4 \pi) \gamma^{\prime-p} P\left(\gamma_{1}, \gamma_{2}, \gamma^{\prime}\right)$, where $\gamma^{\prime}$ is the Lorentz factor of the leptons, $k$ is a constant and $P\left(\gamma_{1}, \gamma_{2}, \gamma^{\prime}\right)=1$ for $\gamma_{1} \leq \gamma^{\prime} \leq \gamma_{2}$, and 0 otherwise.

Using the Lorentz invariant $n / \gamma^{2}$ and the relation $\gamma=$ $D \gamma^{\prime}$ between the Lorentz factor of the electrons/positrons in the lab frame and the blob frame respectively, we get that the electron density in the lab frame is

$n(\gamma)=\frac{k}{4 \pi} D^{2+p} \gamma^{-p} P\left(\gamma_{1} D, \gamma_{2} D, \gamma\right)$,

where $D=[\Gamma(1-\beta \cos \phi)]^{-1}$ is the usual Doppler factor: $\beta$ and $\phi$ are the bulk velocity in units of $c$ and the viewing angle, respectively.

The blob is then injected into an isotropic monoenergetic photon field due to the companion star (typically UV photons) and will then produce the inverse Compton upscattering of some of these stellar photons. With respect to the photon field, the lab frame rate of IC interactions per final photon energy is,

$\frac{\mathrm{d} N_{\mathrm{p}}}{\mathrm{d} t \mathrm{~d} \epsilon}=\frac{3 \sigma_{\mathrm{T}} c}{4 \epsilon_{0} \gamma^{2}} f(x)$

where $\sigma_{\mathrm{T}}$ is the Thomson cross section. For the case of Thomson scattering and assuming isotropic scattering in the electron frame (Georganopoulos et al. 2001b),

$f(x)=\frac{2}{3}(1-x) P\left(1 / 4 \gamma^{2}, 1, x\right), \quad x=\frac{\epsilon}{4 \gamma^{2} \epsilon_{0}}$.

The luminosity is then obtained by integrating the scattering rate (2) over the particle energy distribution (that can be deduced using the density distribution given in Eq. (1) and the volume of the blob), and multiplying by the observed photon energy $\epsilon m_{\mathrm{e}} c^{2}$ and the number density $n_{\mathrm{p}}=U / \epsilon_{0} m_{\mathrm{e}} c^{2}$ (where $U$ is the energy density of the photon field). When $p$ is steeper than 1 and $\gamma_{\min }=\left(\epsilon / 4 \epsilon_{0}\right)^{1 / 2} \ll \gamma_{\max }=\gamma_{2} D$, the luminosity reduces to:

$L_{\epsilon}=\frac{\mathrm{d} L}{\mathrm{~d} \epsilon \mathrm{d} \Omega} \approx D^{3+p} \frac{k V \sigma_{\mathrm{T}} c U 2^{p-1}}{\pi \epsilon_{0}(1+p)(3+p)}\left(\frac{\epsilon}{\epsilon_{0}}\right)^{-(p-1) / 2}$.

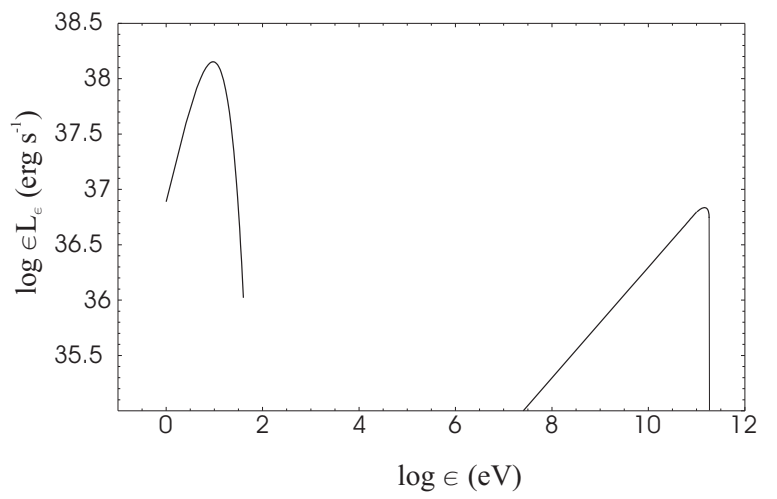

Fig. 1. Spectral high-energy distribution of the scattered photons for a microquasar injecting a power-law spectrum of electrons in the photon field of the high-mass stellar companion (an O7 star in this example). A cut off at Lorentz factors $\gamma \sim 10^{3}$ has been assumed. The spectral energy distribution of the star is also shown (left top corner).

In the Klein-Nishima regime numerical integrations are required (see Georganopoulos et al. 2001b). The important point is that if $\gamma_{2} \sim 10^{3}$ then luminosities of $\sim 10^{36-37} \mathrm{erg} \mathrm{s}^{-1}$ can be obtained in the observer's frame at EGRET's energy range (100 MeV-20 GeV).

However, in the case of a single blob, the interaction time with the photon field, and hence the duration of the gamma-ray flare, will be strongly limited by the apparently superluminal speed of the feature. For instance, in the case of an O7 star and a blob with $\beta=0.98(\Gamma=5)$, the flux will decrease to $1 / e$ of its original value in $\sim 1 \mathrm{~s}$. Gamma-ray production by blobs in microblazars are then transient phenomena difficult to detect on Earth. This is not the case, however, of microblazars with persistent jets.

For a continuous relativistic flow the amplification due to the Doppler factor changes from $D^{3+p}$ to $D^{2+p}$ in Eq. (4) (Lind \& Blandford 1985). In this case the external Compton scattering of stellar photons yields a stable source inasmuch as the flux is not perturbed. We have calculated the spectral energy distribution for a specific model with $p=2$, bulk Lorentz factor $\Gamma=5$, viewing angle $\phi=10 \mathrm{deg}$, photon field of an O7 star (photon energies $\sim 10 \mathrm{eV}$ ), and a high-energy cut off of $\gamma_{2}=10^{3} \gg \gamma_{1}$. The results are shown in Figure 1, where we also show the energy distribution of the stellar photons. We see that luminosities of $\sim 10^{36-37} \mathrm{erg} \mathrm{s}^{-1}$ can be obtained at $E>100 \mathrm{MeV}$. Further details can be introduced through a broken particle spectrum in orther to take into account synchrotron cooling, but these refinements are not necessary here.

It is interesting to notice that whereas the gammaray emission is amplified by a factor $D^{2+p}=D^{3+2 \alpha}$, the synchrotron jet emission results amplified only by $D^{2+\alpha}$, where $\alpha=(p-1) / 2$ is the synchrotron spectral index (see Dermer 1995). Consequently, "red" microblazars, whose synchrotron spectral energy distribution peaks at IR energies, will not be ultra-luminous X-ray sources (ULXs) since only a small part of the radiated power goes into 


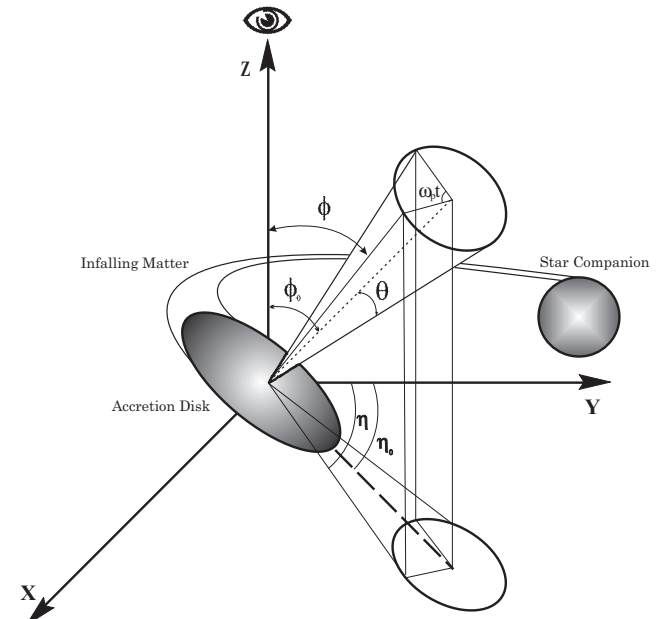

Fig. 2. Precessing jet model.

the X-ray synchrotron tail of their spectra and this emission, in addition, is not so strongly boosted as the inverse Compton gamma-ray emission. Only in case of very low values of $\gamma_{2}$ (say around $10^{2}$ ) we could get an X-ray source $\left(L_{\mathrm{X}} \sim 10^{33-34} \mathrm{erg} \mathrm{s}^{-1}\right)$ without a much more energetic gamma-ray source.

The companion star in a high-mass microblazar system not only provides a photon field for inverse Compton interactions, but also a gravitational field that can exert a torque onto the accretion disk around the compact object. The effect of this torque, in a non-coplanar system, is to induce a Newtonian precession of the disk. If the jets are coupled to the disk, as it is usually thought, then the precession will be transmitted to them. This situation, which should not be confused with the geodetic precession (a purely General Relativity effect), has been extensively studied in the case of SS433 (Katz 1980) and extragalactic sources like 3C273 (e.g. Romero et al. 2000).

A sketch of the situation is presented in Fig. 2. The disk Keplerian angular velocity is $\omega_{\mathrm{d}}=\left(G M / r_{\mathrm{d}}^{3}\right)^{1 / 2}$, where $M$ is the mass of the compact object and $r_{\mathrm{d}}$ is the radius of the precessing part of the disk. The orbital period is $T_{\mathrm{m}}$ and the orbital radius is given by Kepler's law: $r_{\mathrm{m}}^{3}=\left(G / 4 \pi^{2}\right)(m+M) T_{\mathrm{m}}^{2}$, with $m$ the mass of the star.

The angular velocity of the tidally induced precession can then be approximated by $\omega_{\mathrm{p}} \approx-3 G m \cos \theta / 4 r_{\mathrm{m}}^{3} \omega_{\mathrm{d}}$ (Katz 1980; Romero et al. 2000), where $\theta$ is the halfopening angle of the precession cone. We can now introduce a time-parametrization of the jet's viewing angle as (see Abraham \& Romero 1999):

$$
\begin{aligned}
\phi(t)= & \arcsin \left[x^{2}+y^{2}\right]^{1 / 2} \\
x= & \left(\cos \theta \sin \phi_{0}+\sin \theta \cos \phi_{0} \sin \omega_{\mathrm{p}} t\right) \cos \eta_{0} \\
& -\sin \theta \cos \omega_{\mathrm{p}} t \sin \eta_{0} \\
y= & \left(\cos \theta \sin \phi_{0}+\sin \theta \cos \phi_{0} \sin \omega_{\mathrm{p}} t\right) \sin \eta_{0} \\
& +\sin \theta \cos \omega_{\mathrm{p}} t \cos \eta_{0},
\end{aligned}
$$

where all angles are defined in Fig. 2.
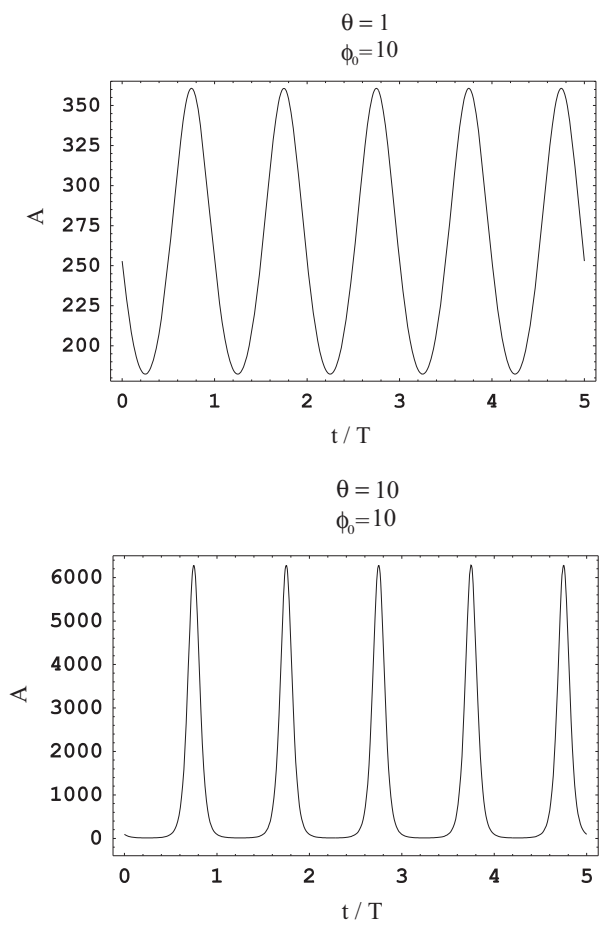

Fig. 3. Variation of the amplification factor for continuous jet emission as a function of time in the precessing microblazar model (for two different opening angles). Time units are normalized to the precessing period.

In Fig. 3 we show the time evolution of the boosting amplification factor of the gamma-ray emission for the case of a continuous jet for two different sets of geometrical parameters (viewing angle of $10 \mathrm{deg}$ and precession half-opening angle of 1 and $10 \mathrm{deg}$ ). Time units are normalized to the precession period $T$. We see that the flux density can change by a factor of $\sim 6 \times 10^{3}$ in a single period. A very weak, otherwise undetected gammaray microblazar, can increase its flux due to the precession and then enter within the sensitivity of an instrument like EGRET, producing a variable unidentified gamma-ray source. The duty cycle (i.e. the fraction of time in which the source is highly variable) in this example is $\sim 0.2 T$, so the source could appear in several EGRET viewing periods. Just to give a feeling of the magnitudes involved, we mention that for a black hole of $4 M_{\odot}$, an O7Ia stellar companion, an orbital period of 10 days, a half-opening angle of $10^{\circ}$ for the precession, and an accretion disk of $\sim 5 \times 10^{11} \mathrm{~cm}\left(\approx 5 \times 10^{-2} \mathrm{AU}\right)$, we get a precession pe$\operatorname{riod} T \sim 100$ days. The source could then be detectable $\sim 70$ days per year. This is consistent with some EGRET observations of highly variable sources (e.g., the case discussed by Punsly et al. 2000).

\section{Discussion}

Taking into account that there exist more than $\sim 130$ high-mass X-ray binaries detected so far (e.g. Liu et al. 2000) and that this number should be a small fraction of the total number of these objects in the Galaxy, it is 
not unreasonable to expect the existence of a few tens of microblazars at mid and low galactic latitudes that could be responsible for the variable galactic gamma-ray sources detected by EGRET. Moreover, the recently discovered X-ray transient V4641 Sgr, which seems to harbor a $\sim 9 M_{\odot}$-black hole and displays extreme superluminal velocities, could be a microblazar (Orosz et al. 2001). Since the stellar companion is a late B-type star, external Compton gamma-ray production is not expected to be very efficient in this case, but its high-energy emission could fall within GLAST sensitivity. Another interesting candidate, from the theoretical point of view, is the high-mass X-ray binary LS I $+61^{\circ} 303$, which presents a one-sided jet at milliarsecond scales and evidence for a precessing accretion disk (Massi et al. 2001). The intrinsic jet velocity, however, seems not to be very high: $\sim 0.4 c$, but the source location is consistent with its identification with a highly variable gamma-ray source 3EG J0241+6103 (Tavani et al. 1998; Torres et al. 2001).

Not all microblazars should be confined to the galactic plane. Recent direct measurements of proper velocities in both low- (Mirabel et al. 2001) and high-mass (Ribó et al. 2002) microquasars show that these objects can present very high velocities and then could be ejected from the galactic plane. This is also supported by the discovery of V4641 Sgr at $b \sim-4.8 \mathrm{deg}$ (estimated distance: $7.4 \leq d \leq 12.3 \mathrm{kpc}$ ). In the case of microquasars with high-mass companions, which are young objects, we could expect to find them up to distances $~ 100$ pc (Ribó et al. 2002) or even more (e.g. V4641 Sgr) from the galactic plane, so they could explain some of the mid-latitude unidentified gamma-ray sources (Grenier 2001). A few low-mass microquasars could have escaped to the galactic halo and consequently might be related to some highlatitude unidentified EGRET detections, if the jets are powerful enough.

Perhaps the best way to identified precessing gammaray microblazars is through the detection of the electronpositron annihilation feature in their spectra. This annihilation signature should appear as a broad, blueshifted (by a factor $D$ ) line in the spectrum at a few $\mathrm{MeV}$, exactly within the energy range of IBIS imager of the forthcoming INTEGRAL satellite. Due to the precession of the jet, the Doppler factor will change periodically with time, and hence the position of the annihilation peak should oscillate in energy in the lab frame around a mean value (for a detailed discussion of the phenomenon see Abraham et al. 2001). Chandra X-ray observations of non-thermal radio sources within the EGRET location error boxes (a complete list of these sources is given by Torres et al. 2001) could help to find candidates to new microquasars (through the detection of X-ray disk emission), and then INTEGRAL exposures could be used in an attempt to find the annihilation line. Such a detection would be a remarkable discovery, since it would establish, at a same time, the matter content of the jets in microquasars, and would help to clarify the nature of some variable unidentified high-energy gamma-ray sources.
Acknowledgements. We are very grateful to M. Georganopoulos for insightful discussions and to the referee, M. Massi, for her valuable comments. I.F.M. acknowledges support from grant PIP 0049/98 and Fundación Antorchas. G.E.R. is supported by the research grants PICT 03-04881 (ANPCT) and PIP 0438/98 (CONICET), as well as by Fundación Antorchas. He is very grateful to staff of the Max Planck Institut für Kernphysik at Heidelberg, where part of his research for this project was carried out.

\section{References}

Abraham, Z., \& Romero, G. E. 1999, A\&A, 344, 61

Abraham, Z., Romero, G. E., \& Durouchoux, P. 2001, ESA SP-459, 131

Aharonian, F. A., \& Atoyan, A. M. 1998, New Astron. Rev., 42,579

Dermer, C. D. 1995, ApJ, 446, L63

Georganopoulos, M., Aharonian, F. A., \& Kirk, J. G. 2001a, A\&A, submitted [astro-ph/0110379]

Georganopoulos, M., Kirk, J. G., \& Mastichiadis, A. 2001b, ApJ, 561, 111

Gehrels, N., Macomb, D. J., Bertsch, D. L., et al. 2000, Nature, 404,363

Grenier, I. A. 2000, A\&A, 364, L93

Grenier, I. A. 2001, in The Nature of Unindentified Galactic Gamma-Ray Sources, ed. A. Carraminana, O. Reimer, \& D. Thompson (Kluwer Academic Publishers, Dordrecht), 51

Hartman, R. C., Bertsch, D. L., Bloom, S. D., et al. 1999, ApJS, 123, 79

Katz, J. I. 1980, ApJ, 236, L127

Lind, K. R., \& Blandford, R. D. 1985, ApJ, 295, 358

Liu, Q. Z., van Paradijs, J, \& van den Heuvel, E. P. J. 2000, A\&AS 147, 25

Massi, M., Ribó, M., Paredes, J. M., et al. 2001, A\&A, 376, 217

Mirabel, I. F., \& Rodríguez, L. F. 1999, ARA\&A, 37, 409

Mirabel, I. F., Dhawan, V., Mignani, R. P., et al. 2001, Nature, 413, 139

Orosz, J. A., Kuulkers, E., van der Klis, M., et al. 2001, ApJ, 555,489

Paredes, J. M., Martí, J., Ribó, M., \& Massi, M. 2000, Science, 288, 2340

Punsly, B., Romero, G. E., Torres, D. F., \& Combi, J. A. 2000, A\&A, 364, 552

Ribó, M., Paredes, J. M., Romero, G. E., et al. 2002, A\&A, in press

Romero, G. E., Benaglia, P., \& Torres, D. F. 1999, A\&A, 348, 868

Romero, G. E., Chajet, L., Abraham, Z., \& Fan, J. H. 2000, A\&A, 360, 57

Romero, G. E. 2001, in The Nature of Unindentified Galactic Gamma-Ray Sources, ed. A. Carraminana, O. Reimer, \& D. Thompson (Kluwer Academic Publishers, Dordrecht), 65

Romero, G. E., Torres, D. F., Benaglia, P., et al. 2001, ESA SP-459, 485

Tavani, M., Kniffen, D., Mattox, J. R., et al. 1998, ApJ, 497, L89

Torres, D. F., Romero, G. E., Combi, J. A., et al. 2001, A\&A, 370,468 